\title{
Low-parametric Induced Current - Magnetic Resonance Electrical Impedance Tomography for Quantitative Conductivity Estimation of Brain Tissues using A Priori Information: A Simulation Study
}

\author{
Nele De Geeter, Guillaume Crevecoeur, and Luc Dupré
}

\begin{abstract}
Accurate estimation of the human head conductivity is important for the diagnosis and therapy of brain diseases. Induced Current - Magnetic Resonance Electrical Impedance Tomography (IC-MREIT) is a recently developed non-invasive technique for conductivity estimation. This paper presents a formulation where a low number of material parameters need to be estimated, starting from MR eddy-current field maps. We use a parameterized frequency dependent 4-Cole-Cole material model, an efficient independent impedance method for eddy-current calculations and a priori information through the use of voxel models. The proposed procedure circumvents the ill-posedness of traditional IC-MREIT and computational efficiency is obtained by using an efficient forward eddy-current solver.
\end{abstract}

\section{INTRODUCTION}

The accurate quantification of the human head conductivity values is important for the diagnosis and the therapy of neurological diseases. Accurate conductivity estimation will enhance the resolution of EEG/MEG source localization and imaging [1] and the design of therapy devices, e.g. Transcranial Magnetic Stimulation (TMS) where the conductivity value has a significant influence on results [2]. Electrical Impedance Tomography (EIT) is a medical imaging technique in which electrical properties as conductivity and permittivity of parts of the body are inferred from boundary measurements [3]. Typically, alternating currents are injected into the subject through conducting electrodes attached to its skin. The resulting electrical potentials are measured and used to reconstruct the impedance distribution. However, large changes in the electrical properties at the interior of the subject lead to small potential changes on the surface of the subject. Due to this ill-posed problem, the spatial resolution of the conventional EIT is limited [4].

In order to increase the resolution, Magnetic Resonance Electrical Impedance Tomography (MREIT) was introduced [5]. While EIT is limited by the measurements of currentvoltage data on the surface of the subject, MREIT utilizes the internal magnetic flux density data obtained using a Magnetic Resonance Imaging (MRI) scanner. By exploiting the relationship between the electrical properties and the magnetic flux density, the conductivity distribution inside the object can be estimated.

N. De Geeter, G. Crevecoeur, and L. Dupré are with the Department of Electrical Energy, Systems and Automation, Ghent University, 9000 Ghent, Belgium, E-mail: Nele.DeGeeter@UGent.be, Guillaume.Crevecoeur@UGent.be and Luc.Dupré@UGent.be
In both EIT and MREIT, currents are injected through surface electrodes. However, these electrical conductors cause problems as the appearance of higher current densities near them and susceptibility artifacts [6]. To avoid these problems, Induced Current - Magnetic Resonance Electrical Impedance Tomography (IC-MREIT) was proposed by Özparlak and İder, similar to induced current EIT [7], whereby currents are induced inside the subject by using external coils [6].

This paper proposes a numerical scheme for solving the IC-MREIT inverse problem, i.e. conductivity estimation.

\section{METHODOLOGY}

In this paper, we focus on obtaining an as accurate as possible estimation of the conductivity values by using the following methodologies: (1) eddy-current induction gradient: In [6], [8], the proposed MR pulse sequence consists of sinusoidal excitation that induce sinusoidal eddy currents. Here, we use a trapezoidal eddy-current induction gradient. In this way, a frequency dependent material model needs to be implemented. We use the 4-Cole-Cole model [9]. (2) a priori information using segmented Tl-weighted MR images as voxel model: A constraint is needed in order to obtain the absolute conductivity values [10]. We use in this paper segmented MR images. In this way, the high number of parameters, used in [6], [8] that needs to be recovered is reduced to a low number so that the ill-posedness of the inverse problem is significantly reduced. (3) time-efficient Independent Impedance Method (IIM) as forward eddycurrent solver: Since a forward eddy-current solver needs to be evaluated many times in an iterative loop for solving the inverse problem, an efficient forward model is needed so to avoid prohibitive computational times. We use a recently developed IIM [11] that is based on the impedance method where acceleration of computations is carried out by solving a linear system of independent equations. (4) iterative scheme for cost function minimization associated to the IC-MREIT inverse problem: The simulated MR phase differences needs to approximate the measured MR phase differences. Since we are using a low-parametric inverse problem, state-of-theart minimization algorithms, here the Nelder-Mead simplex method [12], can be used.

\section{A. The proposed pulse sequence}

By switching magnetic field gradients, eddy currents are induced into the patient under study. To obtain eddy-current field maps, a gradient echo sequence was used, as in [13]. 
This sequence was repeated twice, once with an eddycurrent induction (ECI) gradient switched before the $90^{\circ} \mathrm{RF}$ excitation pulse and once in the absence of this ECI gradient. The phase images from both signals were recorded and phase unwrapped. The eddy-current field map was then obtained by taking a pixelwise subtraction of both phase images. The ECI gradient, as shown in Fig. 1, can be changed with respect to its gradient amplitude, gradient time and the delay between gradient and excitation pulse. Moreover, the gradient can be applied in any imaging direction and combinations of these. The rate of ascent and descent of the trapezoidal gradient is set to the optimal possible slope so to create eddy currents as strong as possible.

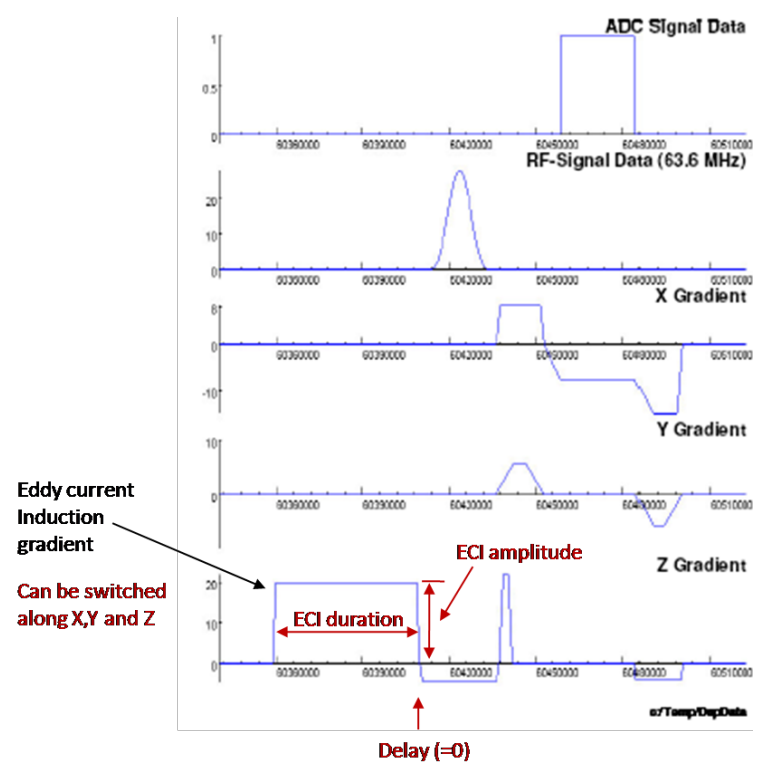

Fig. 1. The proposed gradient echo sequence diagram. In this paper, the ECI gradient is applied in the $z$-direction. The gradient amplitude is 20 $\mathrm{mT} / \mathrm{m}$, the time of descent of the pulse is approximately $100 \mu \mathrm{s}$ and there is no delay.

\section{B. Forward model}

1) Parameterized material model: The 4-Cole-Cole model [9] expresses the frequency dependent complex relative permittivity $\varepsilon_{\mathrm{r}}^{*}$ and conductivity $\sigma$. The spectrum of each biological tissue is characterized by three main relaxation regions $\alpha, \beta$ and $\gamma$ at low $(n=4)$, medium $(n=3)$ and high $(n=2)$ frequencies, and an other minor $\delta$ dispersion $(n=1)$ :

$$
\varepsilon_{\mathrm{r}}^{*}(\omega)=\varepsilon_{\infty}+\sum_{n=1}^{4} \frac{\triangle \varepsilon_{n}}{1+\left(j \omega \tau_{n}\right)^{1-\alpha_{n}}}+\frac{\sigma_{\mathrm{i}}}{j \omega \varepsilon_{0}}
$$

with $\omega$ the angular frequency, $j$ the imaginary unit and the material parameters $\triangle \varepsilon_{n}, \tau_{n}$ and $\alpha_{n}$ chosen appropriate to each tissue. $\varepsilon_{\infty}$ is the permittivity in the high frequency limit and $\sigma_{\mathrm{i}}$ is the static ionic conductivity. Having calculated the complex relative permittivity $\varepsilon_{\mathrm{r}}^{*}=\varepsilon_{\mathrm{r}}^{\prime}-j \varepsilon_{\mathrm{r}}^{\prime \prime}$, the conductivity of each tissue can be computed from the imaginary part:

$$
\sigma(\omega)=\omega \varepsilon_{0} \varepsilon_{\mathrm{r}}^{\prime \prime}(\omega)
$$

with $\varepsilon_{0}=8.8542 \cdot 10^{-12} \mathrm{~F} / \mathrm{m}$ the permittivity in vacuum. In this paper we assume isotropic material properties.
Fig. 2 illustrates the 4-Cole-Cole model applied to brain tissue [9]. The ECI gradient mainly consists of low frequencies. This results in a reduction of the frequency range of interest to $10^{1}-10^{4} \mathrm{~Hz}$, corresponding with the $\alpha$ relaxation ( $n=4)$ in material model (1). Moreover, the value for $\varepsilon_{\infty}$ will be fixed at 2.5 or 4 for low and high water-content tissues respectively [9]. The number of parameters that need to be recovered decreases: the conductivity of each tissue is modelled by the 4 parameters $\triangle \varepsilon_{4}, \tau_{4}, \alpha_{4}$ and $\sigma_{\mathrm{i}}$.

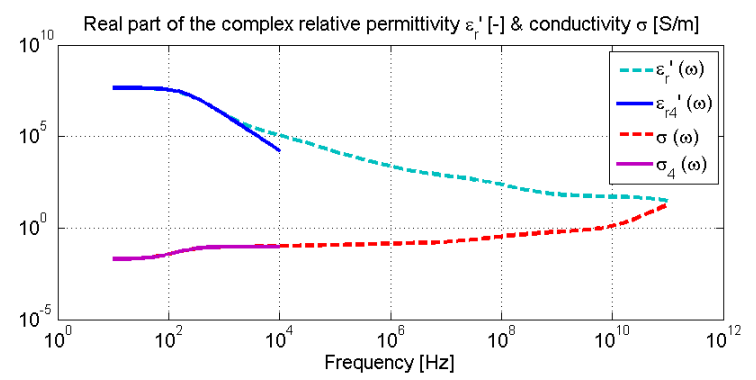

Fig. 2. 4-Cole-Cole model applied to the brain tissue (grey matter).

2) IIM: The 3D Impedance Method (IM) has been widely applied for simulating the induced eddy currents in human bodies [14]. The method discretizes the geometry into regular mesh elements (voxels) and assigns material properties, represented as impedances $\mathbf{Z}$, to each voxel. This leads to a 3D network of impedances with the time-varying magnetic induction $\mathbf{V}$ in each voxel as source model. For our application, IM has the advantage to start from patient-specific T1weighted MR segmented images. However, the linear system of equations $\mathbf{Z} \cdot \mathbf{J}_{\text {eddy }}=\mathbf{V}$ that have to be solved in IM, can be significantly ill-conditioned, leading to a poor numerical convergence or even in some situation no solution at all [15]. In this paper, we use a recently developed IIM [11], based on IM, whereby a set of independent equations is identified by defining independent loops in the $3 \mathrm{D}$ circuit. This results in a reduction of the number of equations that have to be solved, to the benefit of memory burden and computational time. Moreover, the problem is now well-conditioned.

3) Calculation of phase differences: An MR image is the result of a complicated interplay between different magnetic fields: 1) the main DC magnetic field $\mathbf{B}_{\mathrm{DC}}$ of the 3 Tesla MRI scanner in the $z$-direction. 2) three trapezoidal field gradients $\mathbf{G}_{\mathrm{x}}, \mathbf{G}_{\mathrm{y}}$ and $\mathbf{G}_{\mathrm{z}}$ : The $x$-gradient is used for frequencyencoding and readout, the $y$-gradient for phase-encoding and the $z$-gradient for slice selection. These magnetic field gradients cause $\mathbf{G}_{\mathrm{x}} x+\mathbf{G}_{\mathrm{y}} y+\mathbf{G}_{\mathrm{z}} z$ in the $z$-direction for spatial encoding. 3) the RF field $\mathbf{B}_{\mathrm{RF}}$ of the order of $\mathrm{mT}$ for excitation: The source current density $\mathbf{J}_{\text {coil }}$ in the excitation coil will produce a primary magnetic flux density $\mathbf{B}_{\mathrm{p}}$. 4) in this paper, an additional trapezoidal eddy-current induction gradient $\mathbf{G}_{\mathrm{ECI}}$ of the order of milliT/m is used. The latter magnetic field will induce eddy-current densities $\mathbf{J}_{\text {eddy }}$ into the subject, which, on their turn will cause a second magnetic flux density $\mathbf{B}_{\mathrm{s}}$ through Biot-Savart's law:

$$
\mathbf{B}_{\mathrm{s}}(\mathbf{r}, \omega)=\frac{\mu_{0}}{4 \pi} \iiint_{\text {volume }} \frac{\mathbf{J}_{\text {eddy }}(\mathbf{r}, \omega) \times\left(\mathbf{r}-\mathbf{r}^{\prime}\right)}{\left\|\mathbf{r}-\mathbf{r}^{\prime}\right\|^{3}} \cdot d \mathbf{v}^{\prime}
$$


where $\mathbf{r}=(x, y, z)$ and $\mathbf{r}^{\prime}=\left(x^{\prime}, y^{\prime}, z^{\prime}\right)$ refer to field and source points respectively. $\mu_{0}=4 \pi \cdot 10^{-7} \mathrm{H} / \mathrm{m}$ is the permeability in vacuum. The phase obtained with a gradient echo sequence can be calculated as follows:

$$
\phi(\mathbf{r})=\int_{0}^{\mathrm{TE}} \gamma_{\mathrm{g}} \mathbf{B}_{\mathrm{tot}}(\mathbf{r}, t) \cdot d t
$$

with $\gamma_{\mathrm{g}}=26.75 \cdot 10^{7} \mathrm{rad} / \mathrm{sT}$ the gyro-magnetic ratio and the echo time TE being the time between the $90^{\circ} \mathrm{RF}$ pulse and (the center of) signal sampling. The eddy-current field map can be obtained by taking the difference of two phase images; one without the ECI gradient and one with the ECI gradient switched. The phase difference is related to the eddy-current induced field $\mathbf{B}_{\mathrm{s}}$ as:

$$
\Delta \phi(\mathbf{r})=\int_{0}^{\mathrm{TE}} \gamma_{\mathrm{g}} \mathbf{B}_{\mathrm{s}}(\mathbf{r}, t) \cdot d t
$$

Also the imaging gradients will induce eddy currents but these can be assumed to be equal in both experiments, thus cancelling out in the phase difference.

Note that the phase needs to be integrated over time (Eq. 4) while calculations are carried out in the frequency domain. An inverse Fourier transformation is thus needed. Hereby the number of harmonics that is taken into account for the Fourier transformation of the trapezoidal ECI gradient field is important. The accuracy of the results will increase with increasing number of harmonics but so will the computational burden. See Fig.3. Here, 10 harmonics are used in the computations which preserve the accuracy of the solution.

\section{Inverse problem}

We group the material parameters in the vector $\mathbf{p}$, which is characterized as $\mathbf{p}=\left[\Delta \varepsilon_{4}, \tau_{4}, \alpha_{4}, \sigma_{\mathrm{i}}\right]$ for one tissue. For $b$ tissues, p becomes $b \times 4$ dimensional. We define the following IC-MREIT cost function per voxel as:

$$
\mathscr{Y}(x, y, z, \mathbf{p})=\|\Delta \phi(x, y, z, \mathbf{p})-\Delta \varphi(x, y, z)\|
$$

with $\|\cdot\|$ the $L_{2}$-norm that measures the distance between the measured $\Delta \varphi(x, y, z)$ and simulated (with parameter $\mathbf{p}$ ) $\Delta \phi(x, y, z, \mathbf{p})$ phase differences. We reconstruct the conductivity parameters by minimizing the total cost function:

$$
\widehat{\mathbf{p}}=\arg \min _{\mathbf{p} \in P} \sum_{x, y, z} \mathscr{Y}(x, y, z, \mathbf{p})
$$

where a summation is made over all the voxels. $P$ is the feasible domain of the material parameters: $0<\Delta \varepsilon_{4}<5 \cdot 10^{7}$, $10^{-3}<\tau_{4}<1.6 \cdot 10^{-2}, 0<\alpha_{4}<2 \cdot 10^{-1}, 10^{-4}<\sigma_{\mathrm{i}}<7$.

$10^{-1}$. For minimization of the proposed cost function, stateof-the-art iterative techniques can be used. Here, the nongradient based optimization Nelder-Mead simplex method is used as minimization method. Fig. 3 illustrates how the ICMREIT inverse problem solver works.

\section{RESULTS AND DISCUSSION}

\section{A. Simulation setup}

The methodologies are analyzed by performing simulation studies. We gradually increase the number of tissues in the human head. The simulation setup is depicted in Fig. 4.

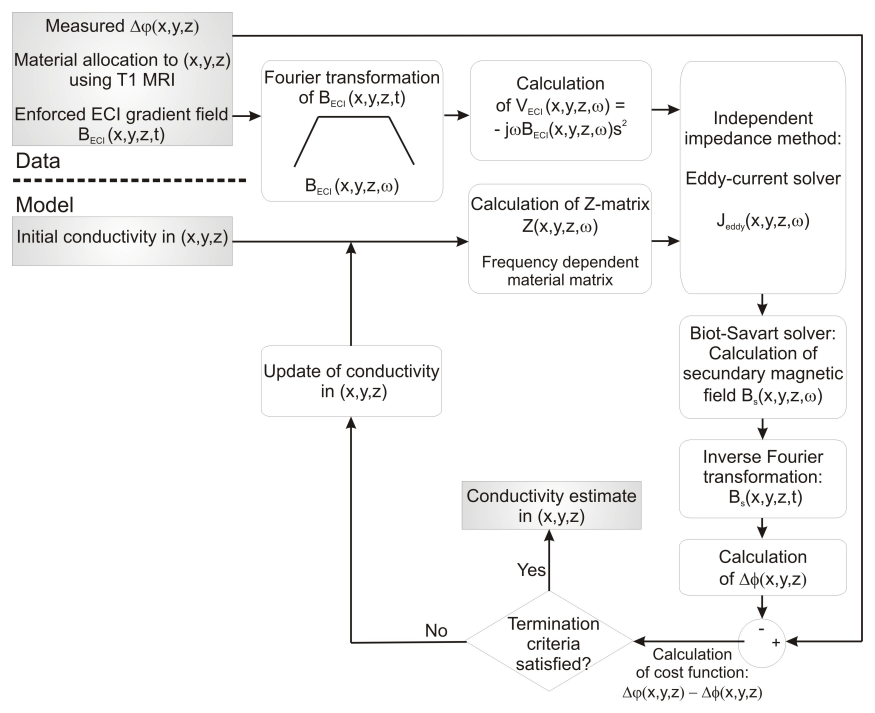

Fig. 3. The flowchart of the IC-MREIT inverse problem solver.

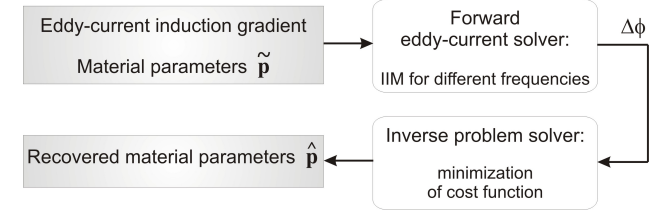

Fig. 4. Setup of simulation studies for validation of methodology.

For a given ECI gradient and known material parameters $\widetilde{p}$, synthetic data $\Delta \phi$ is generated by the forward eddy-current solver and calculation procedures of the phase differences explained in section II.B. The defined low-parametric inverse problem, explained in section II.C is then solved by solving (7). The difference between $\|\widetilde{\mathbf{p}}-\widehat{\mathbf{p}}\|$ expresses the accuracy of the methodology.

This paper is a preliminary study of the proposed methodology and we therefore use a spherical head model with a coarse discretization of $1 \mathrm{~cm}$, as in [6]. The calculation time of one forward model evaluation (with 10 harmonics) is approximately 30 seconds ${ }^{1}$. In this way, the efficiency is obtained and solving the inverse problem, where this forward model is iteratively evaluated, becomes computationally feasible in time in comparison to traditional IC-MREIT [6].

\section{B. Reconstructed conductivity values}

In a first stage, we model the sphere as brain with values $\widetilde{\mathbf{p}}=\left[4.5 \cdot 10^{7}, 0.5305 \cdot 10^{-2}, 0,2 \cdot 10^{-2}\right]$ which correspond with the grey matter given in [9]. Fig. 5 shows the convergence history when solving the inverse problem and the evolution of $\mathbf{p}$ parameters that are evaluated in the iterative loop of the inverse problem. Convergence is obtained after approximately 50 evaluations in the forward solver. This corresponds with a total computational time of $1500 \mathrm{~s}$ or 25 min. Fig. 6 shows the estimated conductivity in the frequency domain which corresponds well with the actual conductivity profile. The value of the low frequency limit $\widehat{\sigma}_{\mathrm{i}}=2.06 \cdot 10^{-2}$ approximates well the actual value $\widetilde{\sigma}_{\mathrm{i}}=2 \cdot 10^{-2}$.

\footnotetext{
${ }^{1} 2$ dual core intel Xuon of $2.0 \mathrm{GHz}$ with $8 \mathrm{~Gb}$ RAM memory on a 64 bit platform
} 

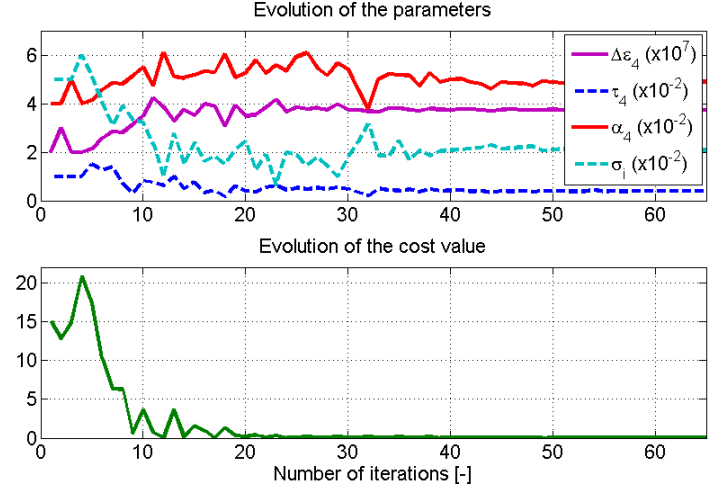

Fig. 5. The evolution of the parameter values and the cost function for the tissue grey matter.

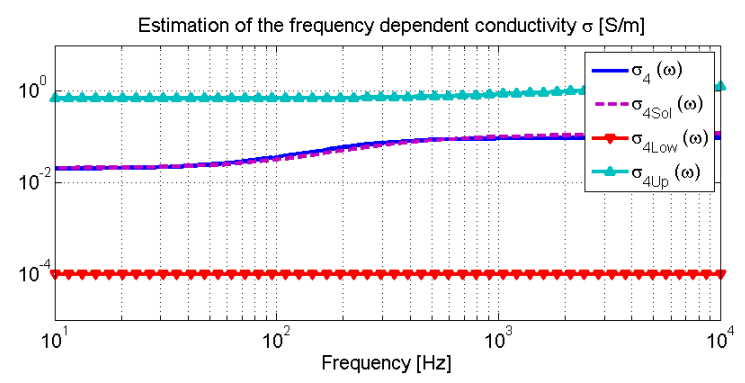

Fig. 6. The estimation of the frequency dependent conductivity of grey matter. The lower and upper bounds are indicated.

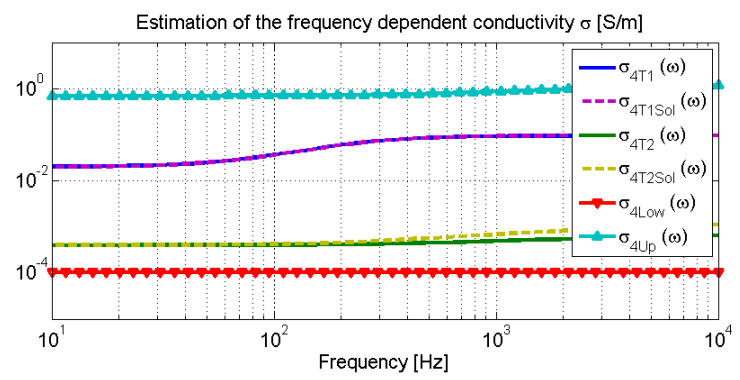

Fig. 7. The estimations of the frequency dependent conductivities of the tissues grey matter and wet skin. The lower and upper bounds are indicated.

In a second stage, we use a spherical model containing the layers brain and scalp. The corresponding parameter values are $\widetilde{\mathbf{p}}=\left[4.5 \cdot 10^{7}, 0.5305 \cdot 10^{-2}, 0,2 \cdot 10^{-2}, 3 \cdot 10^{4}, 0.1592\right.$. $\left.10^{-2}, 0.2,4 \cdot 10^{-4}\right]$ for the tissues grey matter and wet skin according to the 4-Cole-Cole model [9]. Fig. 7 illustrates the estimated conductivity profiles corresponding with a cost of $2.96 \cdot 10^{-5}$. However, these results are obtained with start values close to the actual values. Indeed, the cost function contains many local minima what necessitates the use of a multistart minimization algorithm. This has the disadvantage that conductivity estimation becomes computationally slow, but through the use of parallel computing environment this problem can be resolved.

\section{CONCLUSIONS}

We proposed a methodology for conductivity estimation using IC-MREIT. This methodology consisted of an eddycurrent induction gradient that induces eddy currents at different frequencies and where a conductivity-dependent material model is used. We parameterized the 4-Cole-Cole model in the frequency range $10^{1}-10^{4} \mathrm{~Hz}$ which led to four material parameters that characterize the tissue. A priori information from T1-weighted MR images was used so to keep the inverse problem low-parametric. The simulation studies on a spherical head model showed that it was possible to estimate the conductivity values in the given frequency range. Solving the inverse problem was computationally feasible in time, however, parallelization of these calculations can lead to acceleration in the future. Further research will aim at solving the inverse IC-MREIT problem with noise in the phase differences and real clinical measurements using the proposed methodology.

\section{ACKNOWLEDGMENTS}

The authors gratefully thank Prof. Dr. Ir. Yves De Deene for his help with the gradient echo sequences and the programming on the MRI scanner. They also acknowledge the financial support of GOA07/GOA/006 and IUAP P6/21. G. Crevecoeur is a postdoctoral researcher for the FWO.

\section{REFERENCES}

[1] N.G. Gencer, C.E. Acar, "Sensitivity of EEG and MEG measurements to tissue conductivity", Phys. Med. Biol., vol.49, pp.701-717, 2004.

[2] T.A. Wagner, M. Zahn, A.J. Grodzinsky, and A. Pascual-Leone, "Three-dimensional head model simulation of transcranial magnetic stimulation", IEEE Trans. Biomed. Eng., vol.51, pp.1586-1594, 2004.

[3] P. Metheral, D.C. Barber, R.H. Smallwood, and H.B. Brown, "Three dimensional electrical impedance tomography", Nature, vol.380, pp.509-512, 1996.

[4] A.D. Seagar, D.C. Barber, and B.H. Brown, "Theoretical limits to sensitivity and resolution in impedance imaging", Clin. Phys. Physiol. Meas., vol.8, pp.A13-31, 1987.

[5] E.J. Woo, S.Y. Lee, and C.W. Mun, "Impedance tomography using internal current density distribution measured by nuclear magnetic resonance", Proc. SPIE Ann. Conf., vol.2299, pp.377-385, 1994.

[6] L. Özparlak and Y.Z. İder, "Induced current magnetic resonance electrical impedance tomography", Physiol. Meas., vol.26, pp.S289S308, 2005

[7] N.G. Gençer, M. Kuzuoglu, and Y.Z. İder, "Electrical impedance tomography using induced currents", IEEE Trans. Med. Imag., vol.13, pp. 338-350, 1994.

[8] Y. Liu, S.N. Zhu, and B. He, "Induced current magnetic resonance electrical impedance tomography of brain tissues based on the Jsubstitution algorithm: a simulation study", Phys. Med. Biol., vol.54, pp.4561-4573, 2009.

[9] S. Gabriel, R.W. Lau, and C. Gabriel, "The dielectric properties of biological tissues: III. Parametric models for the dielectric spectrum of tissues", Phys. Med. Biol., vol.41, pp.2271-2293, 1996.

[10] N. Gao, S.A. Zhu, and B. He, "A new magnetic resonance electrical impedance tomography (MREIT) algorithm: the RSM-MREIT algorithm with applications to estimation of human head conductivity", Phys. Med. Biol., vol.51, pp.3067-3083, 2006.

[11] N. De Geeter, G. Crevecoeur, and L. Dupré, "An efficient 3D eddycurrent solver using an independent impedance method for transcranial magnetic stimulation", IEEE Trans. Biomed. Eng., submitted.

[12] J. Nelder and R. Mead, "A simplex-method for function minimization", Comput. J., vol.7, pp.308-313, 1965.

[13] Y. De Deene, C. De Wagter, W. De Neve, and E. Achten, "Artefacts in multi-echo T2 imaging for high-precision gel dosimetry: I. Analysis and compensation of eddy currents," Phys. Med. Biol., vol.45, pp.1807$1823,2000$.

[14] M. Nadeem, T. Thorlin, O.P. Gandhi, and M. Persson, "Computation of electric and magnetic stimulation in human head using the $3 \mathrm{D}$ impedance method", IEEE Trans. Biomed. Eng., vol.50, pp.900-907, 2003.

[15] H. Wang, F. Liu, L. Xia, and S. Crozier, "An efficient impedance method for induced field evaluation based on a stabilized Bi-conjugate gradient algorithm", Phys. Med. Biol., vol.53, pp.6363-6375, 2008. 